\title{
Role of osteodensitometry in osteoporosis and osteopenia in psoriatic arthritis
}

\author{
ILARIE BRIHAN $^{1}$, ANGELICA HĂLMĂJAN ${ }^{2}$, DANIEL BODA ${ }^{3}$, SIMONA LAURA IANOȘI $^{4}$, \\ GYULA LÁSZLÓ FEKETE ${ }^{5}$ and MIHAELA ZDRÎNC $\breve{A}^{6}$
}

\author{
${ }^{1}$ Dermatology Department, Dermatology Clinic, Faculty of Medicine and Pharmacy; \\ ${ }^{2}$ Psychology Department, Faculty of Social-Humanistic Sciences, University of Oradea, 410073 Oradea; \\ ${ }^{3}$ Dermatologic Research Laboratory - National Institute of Diabetes Nutrition and Metabolic Diseases 'N.C. Paulescu', \\ 'Carol Davila' University of Medicine and Pharmacy, 050474 Bucharest; ${ }^{4}$ Dermatology Department, University of Medicine \\ and Pharmacy of Craiova, 200349 Craiova; ${ }^{5}$ Dermatology Discipline, Dermatology Clinic, 'George Emil Palade' University \\ of Medicine, Pharmacy, Science and Technology, 540530 Targu Mures; ${ }^{6}$ Preclinical Disciplines Department,
}

Faculty of Medicine and Pharmacy, University of Oradea, 410073 Oradea, Romania

Received July 8, 2020; Accepted August 7, 2020

DOI: $10.3892 /$ etm.2020.9318

\begin{abstract}
Dual-energy X-ray Absorptiometry (DEXA) determines the mineral contents for the spine, the proximal femur and the entire body. A group of 36 patients with psoriatic arthritis were chosen, with different types of arthropathy, from the total 82 patients with psoriatic arthritis in the study group, for which bone mineral density (BMD) was measured, using the DEXA technique. The chosen study group comprised 12 men, 12 premenopausal women and 12 menopausal women, with psoriatic joint damage and a group of 36 healthy individuals, divided based on the same criteria: Men, premenopausal and menopausal women. This study did not emphasize an association between the patient type and the presented bone demineralization type $\left(\chi_{(2)}^{2}=4.473, \mathrm{P}=0.107\right)$ in men. In premenopausal women, there was a significant association between the patient type and the presented bone demineralization type $\left(\chi_{(2)}^{2}=8.778\right.$, $\mathrm{P}=0.012$ ), as the female patients with psoriatic arthritis presented total and partial demineralization to a larger degree, compared with healthy individuals. In menopausal
\end{abstract}

Correspondence to: Dr Gyula László Fekete, Dermatology Discipline, Dermatology Clinic, 'George Emil Palade' University of Medicine, Pharmacy, Science and Technology, 12 Gheorghe Doja Street, 540530 Targu Mures, Romania

E-mail:dermafek@yahoo.com

Dr Mihaela Zdrîncă, Preclinical Disciplines Department, Faculty of Medicine and Pharmacy, University of Oradea, 10 Piata 1 Decembrie, 410073 Oradea, Romania

E-mail: mzdrinca@yahoo.com

Key words: psoriatic arthritis, bone mineral density, osteoporosis, osteopenia, DEXA bone densitometry women, this study did not highlight an association between the patient type and the presented bone demineralization type $\left(\chi_{(2)}^{2}=4.523, \mathrm{P}=0.104\right)$. Bone demineralization was significant in all three patient groups, both total and partial, in patients with psoriatic arthritis compared with healthy patients. Bone demineralization manifested as osteopenia, with T-scores ranging from -2.5 to -1 , and not as osteoporosis. Determining B MD by DEXA technique revealed osteopenia to a higher degree within the group of patients with psoriatic arthritis compared with the healthy patients, as well as the absence of osteoporosis in psoriatic arthritis.

\section{Introduction}

Bone mineral density (BMD) modification in psoriatic arthritis still represents a largely widespread issue, being the source of several medical controversies. There are few studies regarding the manifestation of osteopenia, generalized osteopenia and osteoporosis in psoriatic arthritis (1).

Localized and systemic bone loss due to increased activity of osteoclasts is well established in PsA. In contrast, the mechanisms responsible for pathological bone remodeling in PsA remain enigmatic although new candidate molecules and pathways have been identified (2).

The bony skeleton is made of $80 \%$ cortical bone and $20 \%$ trabecular bone. The trabecular bone has an elevated metabolic turnover, due to the high surface-volume ratio, eight times higher than that of the cortical bone.

The BMD variations can be measured earlier in the trabecular bone, as compared with the cortical one. Concerning the spine, the articular vertebrae are mainly composed of trabecular bone, compared with the vertebral plates and the spinal apophyses, made of cortical bone. The trochanteric area of the femur has a bone content that is similar to that resulting from the anteroposterior measurement of the spine, namely trabecular bone, whereas the femoral neck contains predominantly cortical bone. 
There are several techniques used in order to determine the BMD, four of which are currently available. According to the anatomical areas measured, they differ in precision and accuracy: Single photon absorptiometry (SPA); dual ohoton absorptiometry (DPA); dual-energy X-ray absorptiometry (DEXA) and quantitative computed tomography (QCT).

\section{Subjects and methods}

DEXA represents the most modern and widely accepted technique. It determined the mineral content of the spine, the proximal femur and the entire body; it can also be used for measurements in the forearm. The term mineral content describes the mineral quantity within the measured area. This allows the determination of the BMD value, by reporting on the measured surface or volume. Therefore, the BMD determined by absorptiometric techniques does not represent a volumetric density, but an area density, considering the scan is bi-dimensional. The DEXA method is also used to scan the extremities, a technique known as peripheral DEXA (pDEXA).

The irradiation, determined by a DEXA examination, is 0.5 to $5 \mu \mathrm{Sv}$. It varies depending on the type of device used, as well as on the area at which the examination is performed. The irradiation dose corresponds to one tenth of the irradiation area generated by a lung X-ray.

The direct measurement of the spine BMD allows a faster determination of trabecular bone loss in early menopausal women and post-cortisone treatments. The measurement of BMD in the femur may generate errors by different positioning of the bone throughout repeated determinations. The cause of errors, which can lead to increased BMD, are large osteophytes, as well as vertebral bundles; therefore, in order to avoid these sources of error, paralleled standard radiological examination is required.

The results obtained by DEXA are: Bone mineral content (BMC) expressed in grams; bone surface area (area per square centimeter) and BMD expressed in $\mathrm{g} / \mathrm{cm}^{2}$. These values must be interpreted according to the device used, as well as the position and the age of the subject.

For the objective determination of the diagnosis, two scores are currently used:

i) The Z-score represents the difference between the measured value of BMD and the average value of persons of the same age and sex with the examined subject, expressed in standard deviations.

ii) The T-score represents the difference between the BMD, measured for an individual and the average value of a young adult, of the same sex, expressed in standard deviations.

Considering the bone mass and the BMD are relatively stable at the end of growth and upon achieving the peak bone mass, they are used as reference value in expressing the BMD changes in standard deviations.

In 1994, WHO established the BMD values measured by DEXA, which characterizes the diagnosis and severity of osteoporosis, based on the T-score: Normal BMD: T-score $>-1$. Low BMD, with less than a standard deviation from that of a young adult. Osteopenia: <-2.5 T-score >-1. BMD between -1 and -2.5 standard deviations, compared with that of a young adult. Osteoporosis: T-score <-2.5. BMD lower than -2.5 standard deviations, compared with that of a young adult. Confirmed osteoporosis: T-score $<-2.5$ plus fracture. BMD lower than -2.5 standard deviations, compared with that of a young adult, when one or more fractures due to bone fragility occur.

The authors chose a group of 36 patients with psoriatic arthritis, with different types of arthropathy, out of the total 82 patients with psoriatic arthritis in the study lot, for which the BMD was measured, using the DEXA technique.

The chosen study group comprised 12 men, 12 premenopausal women and 12 menopausal women, with psoriatic joint damage and a group of 36 healthy individuals, divided based on the same criteria: Men, premenopausal and menopausal women.

The distribution of the cases in the study group by age was similar precisely so that the age does not represent a factor that could influence the values of bone densitometry, considering that age constitutes an important element in the alteration of BMD and particularly, in the onset of osteoporosis (1).

The study was approved by the Ethics Committee of the Oradea County Emergency Clinic Hospital (Oradea, Romania) (approval nos 1386/2019 and 460/2019, respectively), and written informed consent was obtained from all the patients.

\section{Results and Discussion}

Besides the total demineralizations that affect all three evaluated segments simultaneously: Lumbar spine, left and right femur, patients with psoriatic arthritis also present partial demineralizations, located in one or two of the evaluated segments. Thus, out of the 36 patients with psoriatic arthritis, there are partial demineralizations in five patients $(13.88 \%)$. Of the patients with psoriatic arthritis with partial demineralization, only one has both locations at femoral level (2.7\%), while the rest have a partial demineralization with double localization, in which there is lumbar spine demineralization.

Study of partial bone demineralization in men. In male patients with psoriatic arthritis, there are demineralizations in all three evaluated segments (six patients), representing 50\% of the sample volume. There are partial demineralizations with double localization in three patients (25\%) compared with healthy men, where there is demineralization in all three analyzed segments in two patients (16.7\%) and partial demineralization in two patients $(16.7 \%)$, while the rest presents a normal BMD (Table I) (Fig. 1).

This study did not emphasize an association between the patient type and the presented bone demineralization type $\left(\chi_{(2)}^{2}=4.473, \mathrm{P}=0.107\right)$ in men (Table II).

Study of partial bone demineralization in premenopausal women. In premenopausal female patients with psoriatic arthritis, there are demineralizations in all three evaluated segments (nine patients), representing $75 \%$ of the sample volume. There are partial demineralizations with double localization in two patients (16.66\%) compared with premenopausal female patients, where there is demineralization in all three analyzed segments in three patients $(25 \%)$ and partial demineralization in one patient $(8.66 \%)$, while the rest presents a normal BMD (66.66\%) (Table III) (Fig. 2). 


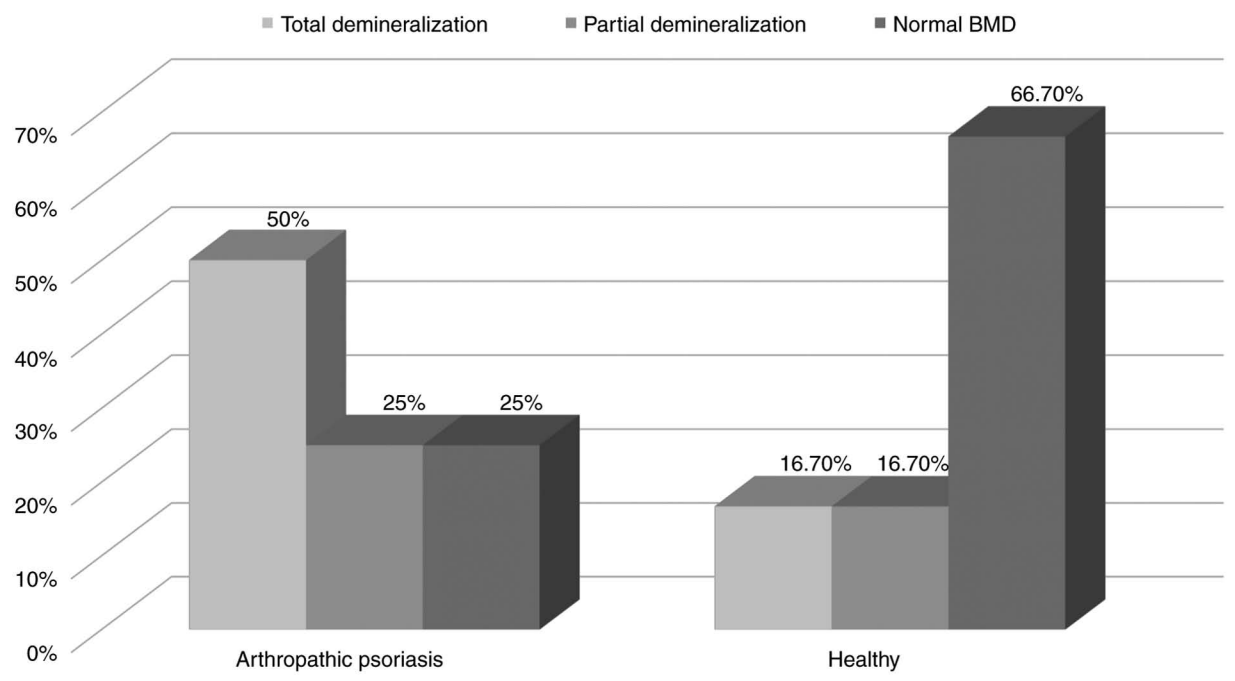

Figure 1. Bone demineralization in men according to the type of patient.

Table I. Chi-square, bone demineralization in men according to the type of patient.

\begin{tabular}{lccc}
\hline & \multicolumn{2}{c}{ Patient type } & \\
\cline { 2 - 3 } $\begin{array}{l}\text { Demineralization } \\
\text { type }\end{array}$ & $\begin{array}{c}\text { Arthropathic } \\
\text { psoriasis }\end{array}$ & Healthy & Total \\
\hline Partial & & & \\
Frequency & 3 & 2 & 5 \\
Expected frequency & 2.5 & 2.5 & 5.0 \\
\% patient type & 25.0 & 16.7 & 20.8 \\
\% total & 12.5 & 8.3 & 20.8 \\
Total & & & \\
Frequency & 6 & 2 & 8 \\
Expected frequency & 4.0 & 4.0 & 8.0 \\
\% patient type & 50.0 & 16.7 & 33.3 \\
\% total & 25.0 & 8.3 & 33.3 \\
No demineralization & & & \\
Frequency & 3 & 8 & 11 \\
Expected frequency & 5.5 & 5.5 & 11.0 \\
\% patient type & 25.0 & 66.7 & 45.8 \\
\% total & 12.5 & 33.3 & 45.8 \\
TOTAL & & & \\
Frequency & 12 & 12 & 24 \\
Expected frequency & 12.0 & 12.0 & 24.0 \\
\% patient type & 100.0 & 100.0 & 100.0 \\
\% total & 50.0 & 50.0 & 100.0 \\
\hline
\end{tabular}

In premenopausal women, there was a significant association between the patient type and the presented bone demineralization type $\left(\chi_{(2)}^{2}=8.778, \mathrm{P}=.012\right)$, as the female patients with psoriatic arthritis present total and partial demineralization to a larger degree, compared with healthy individuals (Table IV).
Table II. Chi-square values for bone demineralization present in men depending on the type of patient.

\begin{tabular}{lccc}
\hline Test & $\chi^{2}$ & df & $P_{\text {bilateral }}$ \\
\hline Pearson's Chi-square & 4.473 & 2 & 0.107 \\
\hline
\end{tabular}

Study of partial bone demineralization in menopausal women. In menopausal female patients with psoriatic arthritis, there are demineralizations in all three evaluated segments (nine patients), representing $75 \%$ of the sample volume. There are partial demineralizations with double localization in three patients (25\%) compared with healthy menopausal female patients, where there is demineralization in all three analyzed segments in four patients (33.33\%) and partial demineralization in one patient $(8.66 \%)$, while the rest presents a normal BMD (Table V) (Fig. 3).

In menopausal women, this study did not highlight an association between the patient type and the presented bone demineralization type $\left(\chi^{2}\left({ }_{2}\right)=4.523, \mathrm{P}=0.104\right)$ (Table VI).

The present study was performed on the three patient groups: Men, premenopausal women and menopausal women, significant modifications regarding the alteration of BMD can be observed in terms of osteopenia in patients with psoriatic arthritis compared with healthy patients.

According to the comparative study of partial demineralizations, the following conclusions were drawn: In the group of men in the study, there is a significant difference of total demineralization between the patients with psoriatic arthritis (50\%) and the group of healthy patients (16.7\%). The difference is insignificant for partial demineralization: $25 \%$ in patients with psoriatic arthritis and $16.7 \%$ in healthy patients.

In the group of premenopausal women, total demineralization was found in $75 \%$ of the patients with psoriatic arthritis, compared with $25 \%$ in the group of healthy patients. Partial demineralization was present in $16.7 \%$ of the patients with psoriatic arthritis, and only $8.3 \%$ in the healthy patients. There is a significant difference regarding total demineralization 


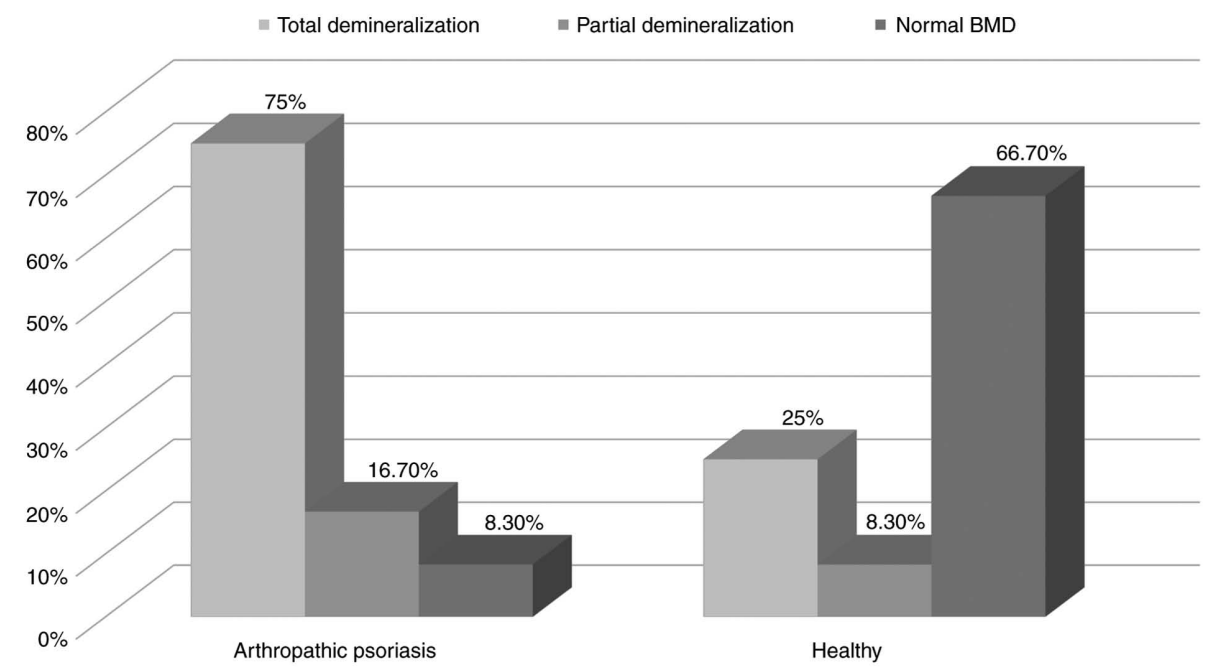

Figure 2. Bone demineralization in premenopausal women according to the type of patient.

Table III. Chi-square, bone demineralization in premenopausal women according to the type of patient.

\begin{tabular}{lccc}
\hline & \multicolumn{2}{c}{ Patient type } & \\
\cline { 2 - 3 } $\begin{array}{l}\text { Demineralization } \\
\text { type }\end{array}$ & $\begin{array}{c}\text { Arthropathic } \\
\text { psoriasis }\end{array}$ & Healthy & Total \\
\hline Partial & & & \\
Frequency & 2 & 1 & 3 \\
Expected frequency & 1.5 & 1.5 & 3.0 \\
\% patient type & 16.7 & 8.3 & 12.5 \\
\% total & 8.3 & 4.2 & 12.5 \\
Total & & & \\
Frequency & 9 & 3 & 12 \\
Expected frequency & 6.0 & 6.0 & 12.0 \\
\% patient type & 75.0 & 25.0 & 50.0 \\
\% total & 37.5 & 12.5 & 50.0 \\
No demineralization & & & \\
Frequency & 1 & 8 & 9 \\
Expected frequency & 4.5 & 4.5 & 9.0 \\
$\%$ patient type & 8.3 & 66.7 & 37.5 \\
\% total & 4.2 & 33.3 & 37.5 \\
TOTAL & & & \\
Frequency & 12 & 12 & 24 \\
Expected frequency & 12.0 & 12.0 & 24.0 \\
\% patient type & 100.0 & 100.0 & 100.0 \\
\% total & 50.0 & 50.0 & 100.0 \\
\hline
\end{tabular}

between the male and female groups, in terms of total demineralization, as it is more frequent in premenopausal women.

In menopausal women, total demineralization was found in $75 \%$ of patients with psoriatic arthritis and in $33 \%$ of healthy patients, while no partial demineralization occurred in women with psoriatic arthritis, compared with the group of healthy patients $(8.3 \%)$. The absence of partial demineralization in the
Table IV. Chi-square values for bone demineralization present in premenopausal women depending on the type of patient.

\begin{tabular}{lccc}
\hline Test & $\chi^{2}$ & df & $\mathrm{P}_{\text {bilateral }}$ \\
\hline Pearson's Chi-square & 8.778 & 2 & 0.012 \\
\hline
\end{tabular}

group of menopausal women with psoriatic arthritis, compared with $16.7 \%$ of the number of premenopausal women with psoriatic arthritis, is explained by the role that menopause plays in accentuating bone demineralization phenomena. Nevertheless, partial demineralization occurs, in the same proportion, in the two groups of women, both before menopause and during menopause, which points to the fact that psoriatic arthritis has an important role in the occurrence of osteopenia, regardless of the status of menopause.

In a study on a group of 18 patients with non-axial psoriatic arthritis and 100 healthy individuals, Frediani et al (3) showed that BMD was significantly lower in subjects with arthritis compared with healthy patients, regardless of sex, age and the status of menopause. Their study showed that demineralization was observed in more than $2 / 3$ of the patients with psoriatic arthritis. This demineralization was not correlated with any signs of inflammation, or with the duration of the disease. However, there is a delayed correlation with age and number of menopausal years.

In a study on a group of 52 patients with peripheral psoriatic arthritis and a control group of 52 healthy individuals, Nolla et al (4) found no significant differences between patients with psoriatic arthritis and control subjects. Nonetheless, in menopausal patients with psoriatic arthritis, a lower BMD of the femoral neck was found, compared with the control group. There were no significant differences in BMD in the lumbar spine in the subgroups of men, premenopausal women and menopausal women (5). The mineral density of the femoral neck was not significantly different in men or in premenopausal women diagnosed with psoriatic arthritis and the control group (3). 


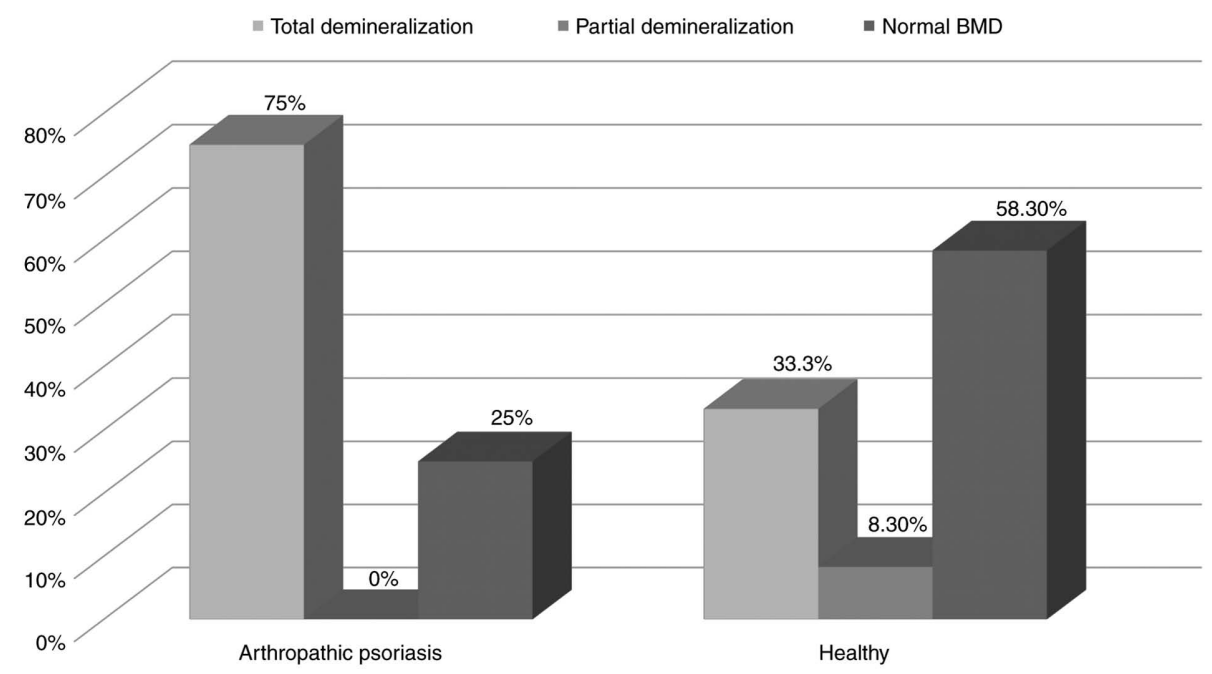

Figure 3. Bone demineralization in menopausal women according to the type of patient.

Table V. Chi-square, bone demineralization in menopausal women according to the type of patient.

\begin{tabular}{lccc}
\hline & \multicolumn{2}{c}{ Patient type } & \\
\cline { 2 - 3 } $\begin{array}{l}\text { Demineralization } \\
\text { type }\end{array}$ & $\begin{array}{c}\text { Arthropathic } \\
\text { psoriasis }\end{array}$ & Healthy & Total \\
\hline Partial & & & \\
Frequency & 0 & 1 & 1 \\
Expected frequency & 0.5 & 0.5 & 1.0 \\
$\%$ patient type & 0.0 & 8.3 & 4.2 \\
$\%$ total & 0.0 & 4.2 & 4.2 \\
Total & & & \\
Frequency & 9 & 4 & 13 \\
Expected frequency & 6.5 & 6.5 & 13.0 \\
$\%$ patient type & 75.0 & 33.3 & 54.2 \\
$\%$ total & 37.5 & 16.7 & 54.2 \\
No demineralization & & & \\
Frequency & 3 & 7 & 10 \\
Expected frequency & 5.0 & 5.0 & 10.0 \\
$\%$ patient type & 25.0 & 58.3 & 41.7 \\
$\%$ total & 12.5 & 29.2 & 41.7 \\
TOTAL & & & \\
Frequency & 12 & 12 & 24 \\
Expected frequency & 12.0 & 12.0 & 24.0 \\
$\%$ patient type & 100.0 & 100.0 & 100.0 \\
\% total & 50.0 & 50.0 & 100.0 \\
\hline
\end{tabular}

In the present study, bone demineralization was significant in all three patient groups, both total and partial, in patients with psoriatic arthritis compared with healthy patients. Bone demineralization manifested as osteopenia, with T-scores ranging from -2.5 to -1 , and not as osteoporosis. Determining BMD by DEXA technique revealed osteopenia to a higher degree within the group of patients with psoriatic arthritis
Table VI. Chi-square $\mathrm{X}^{2}$ values for bone demineralization present in menopausal women depending on the type of patient.

\begin{tabular}{lccc}
\hline Test & $\chi^{2}$ & df & $\mathrm{P}_{\text {bilateral }}$ \\
\hline Pearson's Chi-square & 4.523 & 2 & 0.104 \\
\hline
\end{tabular}

compared with the healthy patients, as well as the absence of osteoporosis in psoriatic arthritis (6,7).

An important factor in modifying BMD is systemic corticosteroid therapy, one of the foundations in inflammatory rheumatism therapy. Treatment with glucocorticoid hormones causes bone loss, because the rate of bone resorption exceeds that of formation. The supraphysiological doses of hormones generate an imbalance by reducing bone formation on the one hand, while suppressing osteoblast function and increasing resorption on the other $(8,9)$. Clinical and densitometric studies on subjects treated with Prednisone $30 \mathrm{mg} /$ day for a long time demonstrated, in $80 \%$ of them, either osteoporosis or the presence of a complication of the same fractures (10-18). Topical treatment with dermocorticoids has created a series of controversies regarding the induced side effects. At skin level, they can generate dermal atrophy with telangiectosis and purpura, promote bacterial, fungal or viral skin infections, induce transient, rosacea and, when applied to the eyelids, can even induce glaucoma and cataract (19). In addition, sudden discontinuing of corticosteroids induces rebound phenomena. The issue of dermocorticoid penetration, which may disrupt adrenal function, has raised discussion. Currently, most studies conclude the pituitary-cortico-adrenal axis is not affected, as well as the absence of the dermocorticoid resonance regarding BMD. Reduced percutaneous absorption and rapid biotransformation within the liver are responsible for the extremely low systemic activity. All of these side effects, induced by systemic and topical cortisone therapy, can be currently counteracted by the introduction, even prophylactic, of classic 
and alternative therapy on cortisone-induced osteopenia and osteoporosis $(20,21)$. Some comorbidity therapies have a negative influence on the evolution of the disease. Beta-blockers used in the treatment of associated cardiological disorders negatively influence the evolution of the disease even in their topical use $(22,23)$. A study, performed on groups of subjects treated with cortisone, shows a three-fold higher incidence of fractures (11).

Nuti et al (12) showed that the best technique for measuring bone density and for assessing the risk of fracture is DEXA.

Osteodensitometry is considered a major indication for the evaluation of patients with long-term corticosteroids (5). Serial bone density measurements by DEXA, every 6-12 months, are recommended when long-term corticosteroids are at doses of at least $7.5 \mathrm{mg} /$ day Prednisone. Thus, it remains an essential method of monitoring corticosteroids in patients with inflammatory rheumatisms. Therefore, osteodensitometry is one of the most important methods for the evaluation of osteoarticular modifications, by decreasing BMD $(18,24)$.

Osteodensitometry: The modification in BMD correlates $75 \%$ with joint disease in psoriatic arthritis. The mineral density was significantly lower, falling in the stage of osteopenia $<-2.5$ T-score $>-1$ in subjects with psoriatic arthritis compared with healthy individuals, without correlating with sex, age or menopause status.

The presence of total bone demineralization, in terms of osteopenia, determined by DEXA technique, on the three levels: Lumbar spine, right femur and left femur, in $75 \%$ of premenopausal women and in the group of menopausal women, demonstrates the lack of correlation of osteopenia with the menopause status in psoriatic arthritis.

\section{Acknowledgements}

Professional editing, linguistic and technical assistance was provided by Irina Radu, Individual Service Provider, certified translator in Medicine and Pharmacy (certificate credentials: Series E no. 0048).

\section{Funding}

No funding was received.

\section{Availability of data and materials}

All data generated or analyzed during this study are included in this published article.

\section{Authors' contributions}

IB was responsible for the clinical management of patients, the processing and scientific interpretation of the data. AH was responsible for the statistical processing of the data and the writing of the manuscript. DB was involved in the data analysis and was responsible for studying and revising the manuscript. SLI was responsible for the analysis of the specialized literature. GLF was responsible for the data processing and revision of the manuscript. MZ was responsible for the statistical data processing, drafting and revising of the manuscript. All authors read and approved the final manuscript.

\section{Ethics approval and consent to participate}

The study was approved by the Ethics Committee of the Oradea County Emergency Clinic Hospital (Oradea, Romania) (approval nos 1386/2019 and 460/2019, respectively), and written informed consent was obtained from all the patients.

\section{Patient consent for publication}

Not applicable.

\section{Competing interests}

The authors declare that they have no competing interests.

\section{Authors' information}

IB-Head of the Department of Dermatology, Emergency Clinical Hospital (Oradea, Romania).

\section{References}

1. Pedreira PG, Pinheiro MM and Szejnfeld VL: Bone mineral density and body composition in postmenopausal women with psoriasis and psoriatic arthritis. Arthritis Res Ther 13: R16, 2011.

2. Paine A and Ritchlin C: Altered bone remodeling in psoriatic disease: New insights and future directions. Calcif Tissue Int 102: 559-574, 2018.

3. Frediani B, Allegri A, Falsetti P, Storri L, Bisogno S, Baldi F, Filippo $\mathrm{P}$ and Marcolongo R: Bone mineral density in patients with psoriatic arthritis. J Reumatol 28: 138-143, 2001.

4. Nolla JM, Fiter J, Rozadilla A, Gomez-Vaquero C, Mateo L, Rodriguez-Moreno J and Roig-Escofet D: Bone mineral density in patients with peripheral psoriatic arthritis. Rev Rhum Engl Ed 66: 457-461, 1999.

5. Chandran S, Aldei A, Johnson SR, Cheung AM, Salonen D and Gladman DD: Prevalence and risk factors of low bone mineral density in psoriatic arthritis: A systematic review. Semin Arthritis Rheum 46: 174-182, 2016.

6. Martinez-Lopez A, Blasco-Morente G, Giron-Prieto MS, Arrabal-Polo MA, Luque-Valenzuela M, Luna-Del Castillo JD, Tercedor-Sanchez J and Arias-Santiago S: Linking of psoriasis with osteopenia and osteoporosis: a cross-sectional study. Indian J Dermatol Venereol Leprol 85: 153-159, 2019.

7. Muñoz-Torres M, Aguado P, Daudén E, Carrascosa JM and Rivera R: Osteoporosis and psoriasis. Actas Dermosifiliogr 110: 642-652, 2019.

8. Ciacli C and Cojocaru M: Systemic osteoporosis - major complication of psoriatic arthritis. Rom J Intern Med 50: 173-178, 2012.

9. Kathuria P, Gordon KB and Silverberg JI: Association of psoriasis and psoriatic arthritis with osteoporosis and pathological fractures. J Am Acad Dermatol 76: 1045-1053.e3, 2017.

10. Riesco M, Manzano F, Font P, García A and Nolla JM: Osteoporosis in psoriatic arthritis: An assessment of densitometry and fragility fractures. Clin Rheumatol 32: 1799-804, 2013.

11. Busquets N, Vaquero CG, Moreno JR, Vilaseca DR, Narváez J, Carmona L and Nolla JM: Bone mineral density status and frequency of osteoporosis and clinical fractures in 155 patients with psoriatic arthritis followed in a university hospital. Reumatol Clin 10: 89-93, 2014.

12. Nuti R, Martini G and Gennari C: Total body, spine, and femur dual X-ray absorptiometry in spinal osteoporosis. Calcif Tissue Int 53: 388-93, 1993.

13. Pfeil A, Krojniak L, Renz DM, Reinhardt L, Franz M, Oelzner P, Wolf $G$ and Böttcher J: Psoriatic arthritis is associated with bone loss of the metacarpals. Arthritis Res Ther 18: 248, 2016.

14. D'Epiro S, Marocco C, Salvi M, Mattozzi C, Luci C, Macaluso L, Giancristoforo S, Campoli M, Scarnò M, Migliaccio S, et al: Psoriasis and bone mineral density: Implications for long-term patients. J Dermatol 41: 783-787, 2014. 
15. Attia EA, Khafagy A, Abdel-Raheem S, Fathi S and Saad AA Assessment of osteoporosis in psoriasis with and without arthritis: Correlation with disease severity. Int J Dermatol 50 : 30-35, 2011.

16. Harrison BJ, Hutchinson CE, Adams J, Bruce IN and Herrick AL: Assessing periarticular bone mineral density in patients with early psoriatic arthritis or rheumatoid arthritis. Ann Rheum Dis 61: 1007-1011, 2002.

17. Lajevardi V, Abedini R, Moghaddasi M, Nassiri SF and Goodarzi A: Bone mineral density is lower in male than female patients with plaque-type psoriasis in Iran. Int J Womens Dermatol 3: 201-205, 2017.

18. Batani A, Brănisteanu DE, Ilie MA, Boda D, Ianosi S, Ianosi G and Caruntu C: Assessment of dermal papillary and microvascular parameters in psoriasis vulgaris using in vivo reflectance confocal microscopy. Exp Ther Med 15: 1241-1246, 2018.

19. Tatu AL, Ionescu MA and Nwabudike LC: Contact allergy to topical mometasone furoate confirmed by rechallenge and patch test. Am J Ther 25: e497-e498, 2018.
20. Tatu AL and Nwabudike LC: Metoprolol-associated onset of psoriatic arthropathy. Am J Ther 24: e370-e371, 2017.

21. Tatu AL, Elisei AM, Chioncel V, Miulescu M and Nwabudike LC: Immunologic adverse reactions of $\beta$-blockers and the skin. Exp Ther Med 18: 955-959, 2019.

22. Nwabudike LC and Tatu AL: Using complementary and alternative medicine for the treatment of psoriasis: A step in the right direction. JAMA Dermatol 155: 636, 2019.

23. Nwabudike LC and Tatu AL: Response to use of complementary and alternative medicine by patients with psoriasis. J Am Acad Dermatol 81: e105, 2019.

24. Grisar J, Bernecker PM, Aringer M, Redlich K, Sedlak M, Wolozcszuk W, Spitzauer S, Grampp S, Kainberger F, Ebner W, et al: Ankylosing spondylitis, psoriatic arthritis, and reactive arthritis show increased bone resorption, but differ with regard to bone formation. J Rheumatol 29: 1430-1436, 2002. 